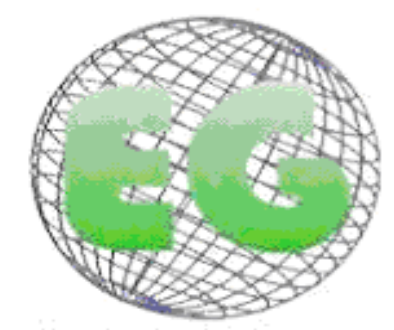

ISSN 1695-6141 N'25.
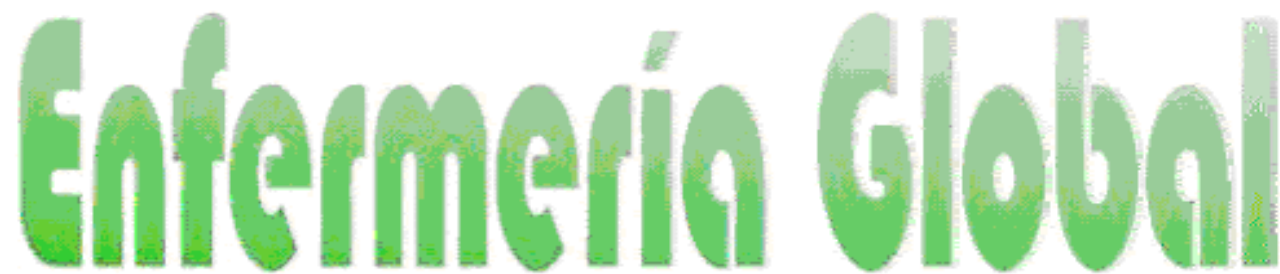

Revista electrónica trimestral de Enfermería

Enero 2012

www.um.es/egloball

\title{
REVISIONES
}

\section{Análisis funcional de los cuidados aplicados a los enfermos de cólera durante la epidemia de 1885 en la ciudad de Murcia, a través de la prensa}

Functional analysis of care applied to those suffering from cholera during the epidemic of 1855 in the town of Murcia, in the press

\section{*García González, J., **Raygal Fernández, PJ., ***Pérez Vigueras, MP., ****Lunaiz Gil, MP., ${ }^{* * * * * J i m e ́ n e z ~ J i m e ́ n e z, ~ M J ., ~ * * * M a r t i ́ n ~ R e t u e r t o, ~ B . ~}$}

*DUE. Máster CC de la Enfermería. Servicio de Urgencias Hospital General Universitario Reina Sofía de Murcia. jaume.garcia@carm.es **DUE. Máster CC de la Enfermería.Servicio de Urgencias de Atención Primaria. Alhama de Murcia. ***DUE. Servicio de Urgencias. Hospital General Universitario Reina Sofía de Murcia. ${ }^{* * * *}$ DUE. Hospital Virgen de las Montañas de Villamantín (Cádiz). ${ }^{* * * *}$ DUE. Supervisora Servicio de Psiquiatría. Hospital Morales Meseguer. Murcia.

Palabras clave: cólera; historia de la enfermería; prensa.

Keywords: cholera; History of nursing; press

\section{RESUMEN}

El estudio tuvo como objetivo conocer, a través de la prensa, los cuidados que se aplicaban a los enfermos durante el brote epidémico de cólera de 1885 en la ciudad de Murcia y quiénes los aplicaban.

La fuente utilizada fue "El Diario de Murcia" del que se revisaron todos los números del día 14 de junio de 1885, fecha en que aparecen los primeros casos, al 9 de octubre de 1885, primer día sin muertes. Los resultados fueron analizados de acuerdo a los postulados del análisis funcional, destacando los profesionales implicados en el cuidado.

Se concluye que la unidad funcional es la Iglesia Católica representada por los valores de las obras de misericordia. El marco funcional es, principalmente, el domicilio aunque se crean hospitales de emergencia para la atención a los enfermos coléricos y los elementos funcionales son el cuidador familiar, en la mayoría de los casos, las religiosas y algunos profesionales (practicantes o enfermeras).

\section{ABSTRACT}

The study had such as an objective to know, via the press, cares that were applied to the sick people during the outbreak epidemic of cholera of 1885 in the city of Murcia and the people who applied the caring them. 
The source use was "Diario de Murcia" of the one which were revised all numbers of the aforementioned daily newspaper of on 14 June 1885, date in which appear first cases, to 9 October 1885, first day without deaths. Results were analysed according to postulates of the functional analysis, emphasising implicated professionals in the care.

We conclude that the functional unit is the Catholic Church represented by the values of works of mercy. The functional framework is primarily the home but are created emergency hospital care for cholera patients and functional elements are the family caregiver, in most cases, religious and some professionals (practitioners or nurses).

\section{INTRODUCCIÓN / JUSTIFICACIÓN}

El cólera representa una amenaza mundial y es un indicador fundamental del grado de desarrollo social. Si bien, actualmente no supone una amenaza ${ }^{1}$, en España durante años fue causante de la muerte de ciudadanos convirtiéndose en un problema de salud pública.

El cólera es una infección aguda causada por el vidrio cholerae que afecta a todo el intestino delgado, caracterizada por diarrea acuosa profusa, vómitos, calambres musculares, deshidratación, oliguria y colapso que puede conducir a la muerte ${ }^{2}$. Se transmite por ingestión de agua, pescado y otros alimentos contaminados por los excrementos de personas portadores, sintomáticos o asintomáticos, de la enfermedad. En las áreas endémicas, los brotes, suelen aparecer durante los meses calurosos. Para controlar la enfermedad es esencial la eliminación correcta de los excrementos humanos, así como la purificación del agua. El agua se debe hervir o clorar antes de beberla, y las verduras y pescados se deben cocinar bien antes de comerlos ${ }^{3}$. Durante los brotes debe hacerse todo lo posible para tratar de identificar a los contactos de los casos y administrar tratamiento a los portadores en fase de incubación ${ }^{2}$.

A finales del siglo XIX los efectos de la infección por cólera tomaban carácter de epidemia en casi toda la geografía española. Esta alteración de la salud pública ha sido ampliamente estudiada en casi todos sus aspectos. Han sido investigadas las polémicas sobre la eficacia de los posibles tratamientos ${ }^{4}$, los datos epidemiológicos ${ }^{5}$, la organización y funciones de los diferentes agentes sociales ${ }^{6}$, los efectos locales de la epidemia ${ }^{7}$, el papel de determinadas personas en la evolución de la epidemia. ${ }^{8}$ A pesar de este exhaustivo análisis, lo que no se encuentra referenciado con suficiente profundidad es el papel que tuvieron los cuidados y los cuidadores en la erradicación de la epidemia. Una de las pocas referencias que señala, a los cuidados enfermeros de la época se hace de manera sucinta y está directamente relacionada con cuidados hospitalarios. Dicha mención se encuentra señalada en el libro conmemorativo de los 100 años de Historia del Hospital de San Juan de Dios, de Antonio Martínez Hernández ${ }^{9}$.

A nivel internacional encontramos, a través del buscador Pubmed, y con los descriptores "epidemic", "cholera" y "nurs*" (con el truncamiento *) unidos por el operador boleano AND, algunos estudios sobre la importancia de las enfermeras en la comunicación de las epidemias ${ }^{10}$, la descripción de las expresiones de las enfermeras en la epidemia del SARS ${ }^{11}$, el papel de las enfermeras del hospital de Mpumalanga como agentes de vigilancia de la infección ${ }^{12}$ y el papel de las enfermeras durante un brote colérico en Kenia ${ }^{13}$.

Contextualizando el tema de la investigación debemos enmarcar los antecedentes históricos en una situación que empieza en septiembre de 1884. Tras recalar en Alicante un barco procedente de Orán se extiende el virus por algunas localidades levantinas llegando hasta el valle del Ebro. La llegada del frío frenó la expansión, pero con los calores de la primavera de 1885 se desarrolló de nuevo y con foco en Játiva se expandió por todo el país ${ }^{7}$. 
Así, en la ciudad de Murcia, a finales del siglo XIX, la situación de insalubridad ${ }^{14}$ la hacía propensa al desarrollo de brotes epidémicos coléricos, por presentar la escasez de agua potable, la ausencia de alcantarillado, la falta de drenaje en zonas semipantanosas. En resumen, ausencia total de infraestructuras sanitarias ${ }^{15}$. A estos factores se adiciona que la ciudad atravesaba desde 1875, una serie de dificultades en la producción agrícola, descenso de la industria sedera y una riada que en 1884 ocasionó un aumento de la pobreza, falta de alimentos y escasez de trabajo ${ }^{14}$.

Algunas de las soluciones propuestas para mejorar las condiciones de la población a nivel de salud e higiene en España contemplaron la realización de una encuesta estatal donde se les preguntaba a los facultativos medidas para mejorar la situación. Dentro de las propuestas que se recogieron se encontraba el aumentar los cauces de saneamiento, drenaje de bancales, limpiar los cauces con aumento de la pendiente 0 plantar árboles ${ }^{14}$. Otra propuesta fue la realización de experimentos con el fin de obtener una vacuna que controlara la epidemia. Un médico de la ciudad de Valencia, el Dr. Ferran, fue pionero en la experimentación de la vacuna, aunque no obtuvo buenos resultados, según algunos autores $^{16}$, pero otros especifican que el médico catalán logró controlar la expansión de la enfermedad inmunizando a la población valenciana ${ }^{8}$. Dejando las controversias a un lado, la cuestión es que la vacuna no llegó a tiempo para evitar la tragedia.

Dadas las condiciones expuestas, el contagio se produjo en una población predispuesta a ello y es por eso que durante este periodo, el cuarto final del s. XIX, hubo dos brotes epidémicos: uno en 1885 y otro, de menor intensidad, en 1890.

En el periodo epidémico de 1885, en la capital murciana y su Huerta, hubo 5015 afectados (Ver tabla I) y 2300 muertos (ver tabla II), lo que equivale a un $27 \%$. Con una razón hombre/mujer 131:100 y con mucha mortalidad infantil que fue imposible de cuantificar, por la dificultad que la en esa época se tenía para censar la población y acceder a los datos. La huerta fue más castigada que la ciudad, y dentro de ella, los barrios proletarios de San Juan y San Antolín ${ }^{14}$.

Tabla I: Individuos enfermos de cólera (1885)

\begin{tabular}{|c|c|c|c|}
\hline Mes & Varones & Hembras & Total \\
\hline Junio & 1697 & 1751 & 3448 \\
\hline Julio & 630 & 763 & 1393 \\
\hline Agosto & 73 & 68 & 141 \\
\hline Septiembre & 9 & 23 & 32 \\
\hline Octubre & - & 1 & 1 \\
\hline Total & 2409 & 2606 & 5015 \\
\hline
\end{tabular}


Tabla II: Individuos fallecidos por el cólera (1885)

\begin{tabular}{|c|c|c|c|}
\hline Mes & Varones & Hembras & Total \\
\hline Junio & 699 & 750 & 1449 \\
\hline Julio & 298 & 299 & 597 \\
\hline Agosto & 33 & 76 & 109 \\
\hline Septiembre & 17 & 35 & 52 \\
\hline Octubre & 2 & 2 & 4 \\
\hline Total & 1093 & 1142 & 2300 \\
\hline
\end{tabular}

\section{Contexto Sanitario en la Ciudad de Murcia durante la época de la epidemia}

En España, a finales del siglo XIX, los establecimientos sanitarios empiezan a ser gestionados por el estado y regulados a través de la Ley de Beneficencia de 1849 y su reglamento de 1852. Esta ley inserta la beneficencia en las administraciones locales por vía municipal y provincial y clasifica los establecimientos en tres:

a) Generales, de carácter nacional para incapacitados o de atención especial como locos, ciegos y sordomudos.

b) Provinciales, cada capital debía tener un hospital, casa de misericordia, de huérfanos, maternidad y expósitos.

c) Municipales, para socorro de necesitados, albergues de mendicidad, hospitalidad en tránsito y beneficencia domiciliaria. ${ }^{17}$

Murcia no era una excepción y durante el periodo de la epidemia existían establecimientos de los tres tipos gestionados por la Junta Local de Sanidad y por la Comisión provincial de beneficencia de la Diputación Provincial. Así, podemos encontrar que la cuidad contaba con los siguientes recursos de atención a los enfermos y necesitados ${ }^{18}$ :

- La Convalecencia. No queda clara en la bibliografía consultada cuál era su función.

- El Asilo de San José. Para el cuidado de ancianos.

- El Manicomio. Para el cuidado de enfermos mentales.

- La Casa de Misericordia y Expósitos. Reservada al cuidado de los huérfanos y abandonados

- El Hospital General o también llamado Hospital de San Juan de Dios. Contaba, aparte de la beneficencia, de la "sala de distinguidos" reservada a las personas pudientes.

Es importante mencionar que la asistencia sanitaria brindada a la población era discriminatoria puesto que las clases acomodadas pagaban a los médicos de familia o de cabecera sus visitas domiciliarias, en tanto los pobres se veían obligados a recurrir a la beneficencia en los hospitales o dispensarios municipales ${ }^{6}$. 
Por tanto, si hacemos un análisis del contexto sanitario en el año de 1885, nos encontramos en un momento previo a la profesionalización de la enfermería que algunos autores como Siles denominan época preprofesional y que otros como Donahue llaman época de influencia aristrocática y militar ${ }^{19}$.

Esta época preprofesional se caracteriza por la división en múltiples estratos de los oficios relacionados con los cuidados: ${ }^{20}$ Barbero, Sangrador, Practicante, Ministrante, Enfermera y Matrona/Partera ${ }^{21}$

Además de esta clasificación, encontramos la realizada por Ventosa ${ }^{22}$, autor que tiene en cuenta las titulaciones que expiden Universidades, Colegios de Cirugía y Escuelas de Medicina: Doctores en Medicina, Doctores en Cirugía, Médicos puros, Físicos, Cirujanos de segunda, Cirujanos latinistas, Licenciados en medicina, Licenciados en cirugía, Bachilleres en medicina, Bachilleres en cirugía, Cirujanos comadrones, Ministrantes y Cirujanos de tercera.

En el año 1861 se presenta una ley de bases para la instrucción pública (Ley Moyano) ofreciéndose una solución para la multitud de oficios presentados en esta época. La ley publicaba un reglamento para la enseñanza de practicantes y matronas que no entró en vigencia, plena, hasta $1868^{23}$.

\section{El papel de la prensa}

La actividad periodística se inicia, en un tiempo en que se hace imprescindible tener un conocimiento de la realidad. Los periodistas surgen como mediadores sociales, como extensiones del conocimiento por nuestra imposibilidad de ubicuidad y por nuestras limitaciones temporales. El periodista mira allí donde no llegan nuestros ojos y nos transmite acontecimientos que pasarán a formar parte de nuestro escenario mental. Él reconstruye claves para interpretar los hechos. El periodismo es un fenómeno de socialización de la experiencia $^{24}$.

Los primeros periódicos murcianos datan del S. XVIII (La Gaceta de Murcia 1706), pero es a partir de la revolución burguesa cuando asumen un papel más definido y se fundan diferentes periódicos: El Semanario Murciano fundado por Antonio Hernández Amores en 1878, El Diario de Murcia, fundado por José Martínez Tornel en 1879 y El Correo de Levante fundado por Pedro Jara Carrillo en $1899^{25}$. El único de estos periódicos que se conserva con todos sus números es el "Diario de Murcia" de Martínez Tornel que es considerado como un costumbrista murciano ${ }^{26}$ y miembro de la ilustración murciana ${ }^{27}$.

La renovación cultural, del antiguo al nuevo régimen, contribuyó a la prensa, con la propagación de ideas políticas y la formación de la opinión pública que cada vez iba adquiriendo más protagonismo. Esta situación se vio beneficiada por la mejora de las comunicaciones, en 1884 se une Murcia y Alicante por ferrocarril, que hacía llegar las noticias de los centros de poder provinciales, las diputaciones, a los pueblos.

En el Diario de Murcia nos encontramos a un predecesor del periodismo actual, hace mención a todo tipo de acontecimientos locales, del más nimio al de mayor alcance y recoge la información nacional de mayor importancia. La archivera municipal de Murcia aconseja a los investigadores que quien quiera " estudiar la vida de Murcia, en el último cuarto de siglo XIX, encontrará en los 28 volúmenes del Diario de Murcia el mayor repertorio de acontecimientos posibles sobre la vida murciana, con todo tipo de detalles y minuciosidad de la época"28. 
El Diario de Murcia tal y como ha sido documentado por distintos autores ${ }^{4,14,18,29}$ se convierte en un instrumento de investigación de los problemas de la sociedad murciana y la realidad histórica, de la época, de primera mano. Es por eso que en este estudio, consideramos importante reconstruir las situaciones que relataba este Diario de los cuidados que se aplicaban a los enfermos durante la epidemia de cólera y conocer quiénes eran los actores que los realizaban.

\section{OBJETIVOS}

- Conocer, a través de la prensa, los cuidados que se aplicaban a los enfermos durante el brote epidémico de cólera de 1885 en la ciudad de Murcia.

- Conocer, a través de la prensa, quiénes dispensaban los cuidados a los enfermos de cólera durante el brote epidémico de cólera de 1885 en la ciudad de Murcia.

\section{MÉTODO}

Se trata de un estudio cualitativo de corte histórico. El tipo de investigación realizada es histórico-social y será conducida a través del análisis documental. El análisis documental consiste en una serie de operaciones destinadas a estudiar y analizar distintas fuentes escritas para comprender la realidad social de los cuidados de enfermería de un determinado momento histórico.

En otras palabras, el análisis documental consiste en examinar documentos históricos, con el fin de investigar los cuidados y poder así reconstruir hechos que nos lleven a conocer cómo evolucionan las prácticas, las profesiones, las condiciones de salud, entre otros factores asociados al cuidado de enfermería.

Para la interpretación de los datos de este estudio se realiza un análisis histórico funcional, el cual consiste en estudiar las funciones e interrelaciones de los distintos elementos y factores implicados en la evolución de la enfermería. Para este tipo de análisis se recomienda realizar un estudio de las estructuras esenciales sobre la que se ha desarrollado la historia. Estas estructuras han sido definidas y catalogadas por Siles ${ }^{21}$ y son:

- La unidad funcional: La estructura o institución social y socializadora básica.

- El marco funcional: Es el lugar, espacio, sitio o emplazamiento donde tienen lugar los fenómenos relacionados con los cuidados.

- El elemento funcional: Actor social responsable de llevar a cabo, directa o indirectamente, el cuidado

En esta investigación se determinarán estos elementos y se presentan en los resultados cuáles son los cuidados aplicados a personas afectadas por la infección colérica en el brote epidémico que azotó a la ciudad de Murcia en el año 1885.

Para llevar a cabo la recogida de datos se realiza la observación documental del periódico "Diario de Murcia". Se revisan todos los números del citado diario del día 14 de junio de 1885, fecha en que aparecen los primeros casos, al 9 de octubre de 1885, primer día sin muertes, un total de 117 días $^{14}$. 
El proceso de análisis se hizo en función del tipo de fenómeno estudiado, es decir, al querer reconstruir históricamente los hechos que marcaban la epidemia del cólera, considerada un problema de salud pública se requiere una clasificación específica de las noticias encontradas en el diario. Por tanto, para la construcción de las categorías se han determinado cuatro aspectos principales de los cuidados relacionados con el continuum salud-enfermedad: ${ }^{30}$

- Cuidados Preventivos ${ }^{31}$ : actividades encaminadas a evitar que aparezca la enfermedad.

- Cuidados Curativos/asistenciales ${ }^{32}$ : Actividades encaminadas al restablecimento de la salud de los enfermos.

- Cuidados post-mortem ${ }^{33}$ : Actividades para la preparación del cadáver y su entierro.

- Cuidados rehabilitadores ${ }^{34}$ : Actividades encaminadas a restablecimiento de las secuelas ocasionadas por la enfermedad.

Se utiliza el microsoft office Excel del paquete de programas de microsoft windows, para realizar una base de datos donde se puede identificar el proceso de análisis de las distintas noticias que hacen referencia al fenómeno de estudio. En esta tabla se tienen los siguientes ítems: fecha, autor, sección del periódico, cuidado (descripción de la noticia), clasificación del cuidado (preventivo, curativo, post-mortem, rehabilitador), unidad funcional, marco funcional y elemento funcional.

Del mismo modo se analizan cuantitativamente las noticias que contengan algunos de los perfiles preprofesionales expuestos por Siles ${ }^{21}$ o Ventosa ${ }^{22}$ descritos en la introducción a éste trabajo. Esta clasificación se plasma en una base de datos de Microsoft Office Excel del paquete de programas de Microsoft Windows, donde se indica: fecha, sección del periódico, nombre del profesional y resumen de la noticia.

\section{LIMITACIONES}

Para el desarrollo de este proyecto de investigación se tuvieron ciertas dificultades inherentes al tipo de estudio desarrollado, un estudio de corte histórico. La primera dificultad a relatar es el acceso restringido a los periódicos, por situarse en archivos específicos del Archivo Municipal de Murcia que exigen de una acreditación como investigador. Estos documentos al estar microfilmados, son de difícil lectura y por tanto, su análisis puede ser lento debido a que se requiere para su tratamiento solicitar fotocopias cuyo coste es elevado y además, por el manejo que se debe realizar a este tipo de documentos, tardan en ser entregadas, aproximadamente, una semana, lo cual retrasa el análisis del investigador.

Durante la realización del trabajo otra limitación encontrada es la dificultad para obtener estudios históricos de enfermería que describieran cómo actuaban los profesionales del cuidado durante las situaciones de epidemia. Las únicas referencias se encuentran en manuales generalistas de historia de la enfermería en España o en el mundo. Estas referencias son escuetas y limitadas dado el contexto en el que se realizan.

\section{RESULTADOS}

Se analizan un total de 101 periódicos de 117 días, debido a que el día lunes no se publicaba "El Diario de Murcia". En total se han seleccionado 160 noticias que contienen alguna referencia a cuidados de salud durante la epidemia. Se han construido dos categorías con el fin de ilustrar los datos encontrados. 


\section{Análisis de los cuidados}

En la siguiente gráfica (llustración I) se muestra como se reparten los tipos de cuidados en las noticias del diario:
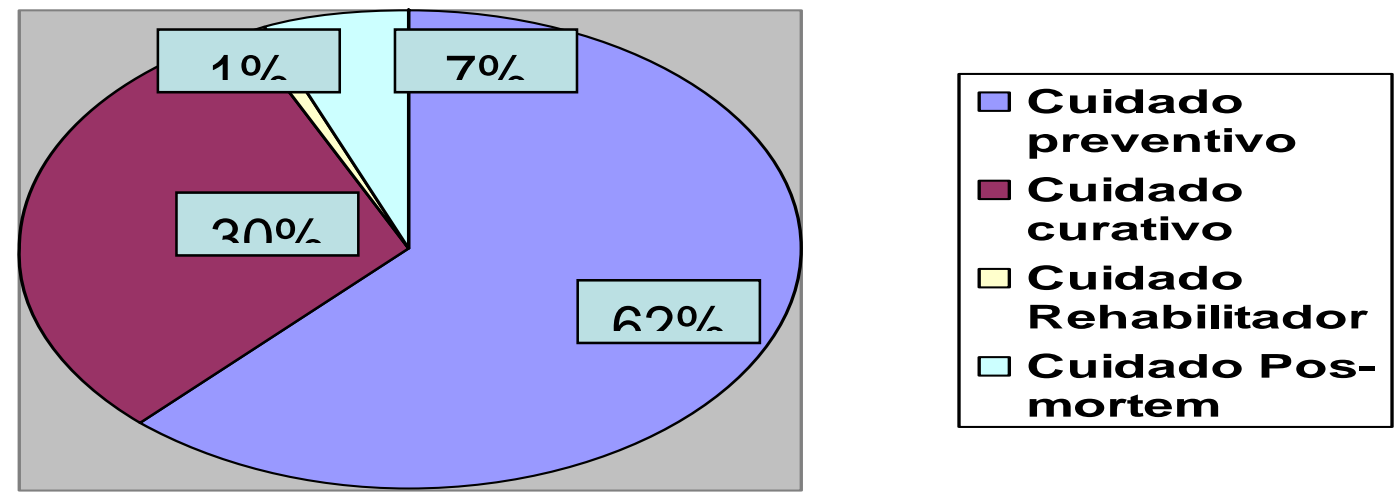

Ilustración 1: Distribución de las noticias según tipo de cuidado

El periódico juega un papel de educador para la salud, pues explica a la población lectora la manera en la que deben prevenir el contagio de la enfermedad. Tal y como lo muestra la gráfica, la mayoría de cuidados aplicados a los enfermos durante el periodo de la epidemia son de carácter preventivo. Dentro de las medidas preventivas destacan las medidas higiénico-dietéticas, algunos ejemplos son los siguientes recortes de prensa:

- "La junta local de sanidad ha dispuesto que las parroquiales procedan sin levantar mano al saneamiento de los focos de infección existente en la ciudad". Publicado el 14/06/1885 en la sección de Noticias Locales.

- "Los practicantes estaban ocupados desinfectando dos casas". Publicado el día 16/06/1885 en la sección "Lo del día”.

- "Como se reparten en el Ayuntamiento el ácido frénico y los desinfectantes se dan a todo el que va a pedirlos". Publicada el 18/06/1885 en la sección "Lo del día".

- "Se han formado una brigada de 24 hombres, que se dividirán por la población, únicamente a la fumigación y desinfección". Publicada el 18/06/1885 en la sección Noticias locales.

- "El Sr. Gobernador indicó: Inspeccionar los alimentos, limitar el tiempo de las tabernas, evitar que se beba agua del Segura al menos llevar el convencimiento a todos de que debe cocerse". 16/06/1885. Lo del día.

- "El cólera es una enfermedad grave, peligrosa; pero cuya gravedad y peligro disminuyen mucho, casi desaparecen, si se observa un buen régimen de vida y no se descuidan los primeros síntomas". 29/07/1885 en Noticias Locales. 
- "El abuso de precauciones no deja de ser abuso, y como todos ellos, perjudicial para la salud. Cierto es que no convienen ahora los laxantes, pero la astricción tampoco es conveniente". 23/07/1885 en "Lo del día".

- "Lo que debemos hacer es aumentar, si cabe, todas las medidas de precaución: limpieza de las calles, desinfección de alcantarillas y de todo foco malsano, saneamiento de cauces de aguas muertas, socorro a los enfermos, convalescientes y pobres... en pocas palabras, todo lo que a la higiene pública y privada se refiere". 22/07/1885. Lo del Día.

- "Se recomienda especialmente para las casas donde ha habido un colérico la desinfección de los excusados con: Permanganato de potasa 50gr, Sulfato de hierro 500, 5 litros de agua. Se echa en cuartas partes en cuatro días en los vasos comunes".2/07/1885.

Las medidas curativas, están presentes en menor proporción que las preventivas. Se centran básicamente en la alimentación y en la aplicación de tratamientos, preparados farmacéuticos, inyecciones hipodérmicas o inoculaciones, por parte de los médicos. Algunos ejemplos de los usados en las noticias del Diario de Murcia son:

- "El enfermo no puede comer arroz y tocino, eso es muy bueno para el sano, fuerte y robusto trabajador, pero no para el que padece en el lecho del dolor". Publicado el día 28/06/1885 en Noticias Locales.

- "El epidemiologo murciano, Maestre, visitó ayer a 43 enfermos y aplicó las inyecciones hipodérmicas". Publicado el 15/07/1885 en Noticias Locales.

- "El uso de láudano en dosis de 15 o 20 gotas administrado en el primer periodo de la enfermedad está dando admirables resultados". Publicado el 30/07/1885 en Noticias Locales.

- "De pocos días a esta parte el origen de todas las defunciones coléricas se debe a excesos en las comidas". Publicado el 14/07/1885 en "Lo del día".

En tanto, las medidas rehabilitadoras son las que se mencionan en menos ocasiones entre las noticias. Sólo aparece la siguiente: "Muchos enfermos escapan del primer ataque del cólera pero sucumban luego por falta de ese cuidado delicado y especialísimo que necesita el cuerpo que ha pasado por tan tremenda crisis". Publicado el 28/06/1885 en la sección "Lo del Día".

Por último, los cuidados post-mortem que aparecen referenciados en las noticias básicamente hablan acerca de la escasez de enterradores y aluden de manera particular a aquellas personas que se atrevían a hacerlo. Las noticias también exponen que algunos cadáveres no son atendidos, dando como razones para este hecho la escasa implicación social por el miedo al contagio. Tomemos como ejemplo la noticia publicada el día 14/06/1885 en la sección Lo del Día: "En una casa de tres pisos, ha muerto una joven, y ni los de abajo han subido, ni los de arriba han bajado". 


\section{Análisis funcional}

Con la revisión exhaustiva que se realiza al periódico podemos concluir que la unidad funcional durante la epidemia que tuvo Murcia a finales del siglo XIX es la Iglesia Católica. Este ente representaba el valor de las obras de misericordia que eran organizadas por medio de las juntas parroquiales. Otros organismo que participa es la Junta Local de Sanidad, órgano de sanidad del Ayuntamiento, pero que está ampliamente influenciado por los valores cristianos y que usa las juntas parroquiales para la atención a la población. A pesar de las ideas liberales del consistorio municipal siguen movidos por la concepción cristiana de los cuidados. Cabe destacar que aparecen algunas noticias en las que librepensadores aplican cuidados por filantropía. Estos últimos aparecen en una proporción muy baja. En resumen podemos concluir que, dada su omnipresencia, la estructura o institución social y socializadora básica sigue siendo la Iglesia Católica.

Los espacios dedicados al cuidado (marco funcional) son tres Casas de Socorro establecidas para la ocasión, el Hospital de San Juan de Dios, la Convalecencia, la Casa de Misericordia, el manicomio y el Asilo de San José, aunque básicamente los cuidados se aplican en el domicilio.

El elemento funcional es básicamente el cuidador familiar pero en "El Diario de Murcia" aparecen noticias sobre diferentes profesionales y preprofesionales implicados en los cuidados de los enfermos de cólera.

A continuación se describen los diferentes elementos funcionales (actores sociales responsables de llevar a cabo, directa o indirectamente, el cuidado) y se hace referencia a las veces que aparecen referenciados en el periódico analizado.

Entre las personas que se dedican, como seglares no religiosos, al cuidado de los enfermos se encuentra que los nombres más usados son el de enfermero, siempre en masculino, y el de practicante. Se distinguen, entre estos últimos, al practicante mayor.

En relación a los enfermeros aparecen 10 menciones'. Se especifica los primeros días de la epidemia la falta de enfermerosii para un mes más tarde, cuando las defunciones en la ciudad han disminuido, suprimirse sus cargosiii. Observamos, en las noticias y anuncios, ofrecimientos de enfermeros que atienden con carácter privado ${ }^{\text {iv }}$.

Se les elogia su trabajo en dos ocasiones ${ }^{v}$ y se censura en solo una por abandono de los enfermos durante la noche "Se personaron en el hospital a la una de la noche, deseosos de conocer el estado de los enfermos y el esmero con el que se les asiste. Llamaron a los enfermeros y nadie contestó". vi.

El único nombre propio de enfermero que encontramos en las páginas de el Diario es para referirse a Antonio Henarejos, un personaje contradictorio: La primera vez que aparece ${ }^{\text {vii }}$ se dice de él que "todo elogio es poco", la segunda vez viii escribe una carta al Gobernador para que lo cambie de destino a otra zona donde el cólera esté azotando más fuerte y por último aparece la noticia de que Henarejos ha sido expulsado del hospital ${ }^{\text {ix }}$.

En relación a los practicantes, descubrimos que los primeros días se dedican a la desinfección " Los practicantes estaban ocupados desinfectando dos casas" y se diferencian éstos de los enfermeros, dado que al constituir las tres casas de socorro en la ciudad aparecen entre los empleados: dos enfermeros y dos practicantes ${ }^{\mathrm{xi}}$. 
En general se habla correctamente del trabajo que realizan xii , pero en una ocasión aparece una carta anónima publicada quejándose de un practicante ${ }^{\text {xiii }}$.

Nos hablan del cargo de practicante mayor que tiene asignado Don Juan Martínez Nicolás. A través de dos noticias nos describe alguna de sus funciones: comadrón ${ }^{\text {xiv }}$ y albacea de los testamentos ${ }^{\mathrm{xV}}$.

Aparece en una primera noticia otro señor que es calificado como "persona que tiene conocimientos prácticos de medicina" "xi pero que más tarde es nombrado como practicante ${ }^{\mathrm{xvi}}$.

Una noticia que llama la atención es que en los partidos rurales de Murcia, a mitad de la epidemia, solo hay tres practicantes ${ }^{\text {xviii. }}$.

En la siguiente tabla se muestran los cuidadores que forman parte de alguna Orden religiosa y que están referenciados en el "Diario de Murcia".

\begin{tabular}{|c|c|c|}
\hline ÓRDEN RELIGIOSA & $\begin{array}{l}\text { No } \\
\text { NOTICIAS }\end{array}$ & CONTENIDO NOTICIAS \\
\hline $\begin{array}{l}\text { Hermanas de la } \\
\text { Caridad }\end{array}$ & $17^{\mathrm{XIX}}$ & 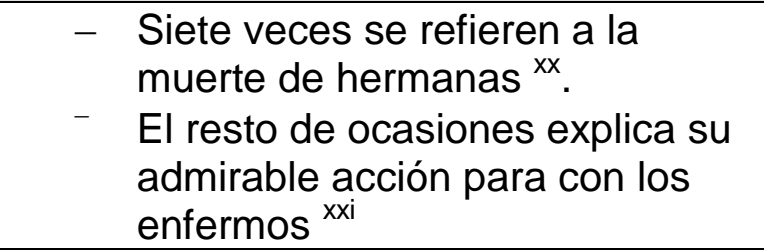 \\
\hline $\begin{array}{l}\text { Hermanitas de los } \\
\text { pobres y las } \\
\text { huérfanas }\end{array}$ & 2 & $\begin{array}{l}\text { - Donación, por benefactores, de } \\
\text { alimentos xxii } \\
\text { - } \quad \text { Muerte de una hermana por } \\
\text { cólera xxiii. }\end{array}$ \\
\hline Siervas de María & 1 & $\begin{array}{l}\text { - Ofrecen sus servicios a los } \\
\text { enfermos de la ciudad, del mismo } \\
\text { modo que lo hacen las hermanas } \\
\text { de la caridad xxiv. }\end{array}$ \\
\hline Padres Jesuítas & 3 & $\begin{array}{l}\text { - Atienden a enfermos de la zona } \\
\text { de Guadalupe } \\
\text { - Muerte de dos hermanos por el } \\
\text { rólera xxv }\end{array}$ \\
\hline
\end{tabular}

Por último, hay una serie de relatos en el diario que hacen mención a un grupo de personas, de distintos oficios/profesiones que se encargan del cuidado de enfermos. La alusión a este tipo de "cuidadores" se encuentra descrita de modo genérico en las noticias del diario. Algunos ejemplos son:

- "Una de esas mujeres que se dedica a la asistencia a los enfermos"xxvi . Esta expresión hace referencia a una mujer que se hacía cargo del cuidado de los enfermos pero a la cual, por lo mencionado en el Diario, le pagaban con las propias ropas de los difuntos. Así hacía referencia a este hecho: "le atribuyeron haberse llevado a casa, las ropas de un colérico difunto, con las que habían pagado sus servicios".

- En la siguiente noticia se observa el hecho de necesitar ayuda de mujeres para el cuidado de los enfermos: xxvii" En vista de las razones expuestas por el director del Hospital San Juan de Dios, se acordó aumentar durante las presentes circunstancias, dos mujeres para que 
ayuden a las hermanas de la caridad en el nuevo manicomio, establecido en la fábrica de puerta nueva"

- Otro relato encontrado nos muestra cómo se describe la situación vivida por un enfermo: Me trajeron a casa y pusieron a mi disposición facultativo y dos hombres, costeados por ellos, a mi cuidado ${ }^{x \times v i i i}$.

- En la siguiente expresión se puede reflejar la situación vivida en la cuidad murciana donde por la epidemia se necesitaba: Gente que practique cuantas operaciones sean necesarias para salvarlo, a parte de los médicos ${ }^{\text {xix }}$.

- Con la siguiente frase encontrada en el diario: se va organizando el hospital todo en cuanto es posible, porque faltan brazos auxiliares ${ }^{x x x}$, se demuestra cómo se constituía un hospital de urgencia para la atención de los enfermos y a su vez, la falta de personal sanitario.

- Otra narración que se hace en la prensa es la de unos jóvenes de reputada ilustración que "Vienen despachando día y noche las medicinas necesarias, bajo la dirección del médico, y acudiendo a las casas de los infectados, a verificar las fumigaciones con un valor incansable y digno de elogio." del cuidado.

- Por último en la sección de anuncios aparecen en varias ocasiones las nodrizas xxxii que forman parte de la historia de nuestra profesión.

\section{CONCLUSIONES}

El ambiente de la ciudad atacada por el cólera está magistralmente descrito, en las hojas del periódico, por su director y propietario Martínez Tornel. En sus secciones "Lo del Día", "Noticias Locales", "Correspondencia" y "Revista Local" describe la evolución de la epidemia. Gracias a este periódico sabemos que la imagen de Murcia recordaba a una ciudad medieval donde estaban presentan la insalubridad y se tenía altos índices de enfermos por cólera

Basándome en los datos analizados en el periódico, se podría afirmar que en el Hospital para enfermos coléricos, la Casa de Misericordia donde se atendían los enfermos de cólera y en el resto de centros asistenciales, sólo se aplicaban cuidados básicos, domésticos, de enfermería para aliviar a los afectados.

En el momento histórico analizado a pesar de que se iniciaban los tratamientos médicos experimentales como las vacunas e inoculaciones, eran solo unos pocos ciudadanos los que podían acceder a ellos, lo hacían en sus domicilios.

Los cuidados básicos para controlar la epidemia de cólera en el año de 1885 consistían en evitar la propagación de la enfermedad y otras medidas higiénicas. Se observa como factor de gran importancia la alimentación, al ser el cólera una enfermedad entérica. La gran preocupación de las instituciones es aportar alimento a los pobres y enfermos, lo cual lo logran con la creación de una cocina económica cuyo acceso era para todos los ciuidadanos...

Otra de las conclusiones a la que podemos llegar es que se puede confirmar la existencia de cuidados enfermeros institucionalizados en enfermedades con gran riesgo para la salud pública y gran incidencia social. Esto es una realidad histórica que puede ir asociada a la 
generación de una profesión puesto que se convierten en un recurso necesario para la población.

Llama la atención que en la mayoría de los datos extraídos en el estudio, los cuidadores profesionales, practicantes o enfermeros, son de género masculino. Este factor podríamos atribuirlo a varias causas;

- La primera es que la situación de "crisis" laboral y del campo hace que los hombres, encargados morales del mantenimiento del hogar, se dediquen a trabajar en todos los ámbitos.

- La segunda es que la situación de alarma por la epidemia haga que los maridos, propietarios de las mujeres, no las dejen trabajar, factor que explicaría la razón de distribución hombre/mujer de 131:100.

- La tercera es que escaseen las mujeres con capacidad para trabajar porque se están dedicando al cuidado de la familia.

Como siempre, la historia de los cuidados y la historia de la enfermería va ligada al rol de género de la mujer.

Podemos concluir que los cuidados, en Murcia y durante la epidemia, los aplican las mujeres en las familias, los practicantes y enfermeros en las instituciones sanitarias y básicamente las religiosas y religiosos que tienen entre sus reglas el cuidado a los enfermos.

Durante la investigación han aparecido algunas dudas en cuanto al análisis del marco funcional. La mayoría de las noticias tienen una connotación religiosa sustentada en las obras de misericordia y en la tradición cristiana, pero nos encontramos ante un Ayuntamiento y un periódico liberal que se contrapone a una oposición conservadora más apoyada en las ideas católicas. A pesar del progreso se puede observar que estamos en un momento de paso entre el marco católico y el marco beneficencia filantrópica.

Para finalizar proponemos una investigación más completa del papel de las enfermeras en la epidemia del cólera de 1885 en la ciudad de Murcia en la que se contemple y analicen todos los archivos disponibles: el del Hospital San Juan de Dios, la Diputación Provincial, la casa de beneficencia y resto de instituciones de la época, incluidos los archivos de las religiosas y religiosos que intervinieron. De la misma manera se podrían incluir el resto de periódicos de la ciudad en el periodo.

\section{BIBLIOGRAFÍA}

1. Cirera L, Cruz O, Martínez C, Madrigal E, Salmerón D, Navarro C. Estadísticas básicas de mortalidad en la Región de Murcia en 2007. Murcia: Consejería de Sanidad y Consejería de Economía Empresa e Innovación; 2008.

http://www.carm.es/econet/sicrem/PU mortalidadBasica/

2. Keryb A. (ed) El Manual Merck. Harcourt. Madrid: 1999.

3. Cólera. Manual Harrisononline en Español. McGraw-Hill Companies. 2006. Disponible en: http://www.harrisonmedicina.com/popup.aspx?alD=71509\&print=yes Consultado el: 21/03/09.

4. Marset P, Sáez JM, Crespo F. El cólera de 1885 y las polémicas doctrinales en la prensa. Revista de la Sociedad Española de Historia de las Ciencias y de las Técnicas, Vol. 20, № 38, 1997 , pags. $273-292$ 
5. Ayala JA. Aspectos sociales de la epidemia del cólera de 1834 en Murcia. En Torres J, Casal F, Mula AJ, Ayala JA y Marset P. De Historia medica murciana. II Las epidemias. Academia Alfonso X el Sabio. Murcia: 1982.

6. López J.M.: "Historia de la Medicina". Ed. HISTORIA 16. Madrid. 1990.

7. Fernández JJ. El Cólera de 1885 en la provincia de Guadalajara. Disponible en: http://biblioteca2.uclm.es/biblioteca/CECLM/ARTREVISTAS/Wad/wad23 fernandezcolera.pd f Consultado el 16/06/2010.

8. Lafuente S, Vilella A, Serrano B, González R, Bruni L. El Cólera. Enf Emerg 2006; 8 (1):10-15. Disponible en: http://www.nexusediciones.com/pdf/ee2006 1/s-8-1-002.pdf Consultado el 21/5/10.

9. Martínez A. Historia del Hospital San Juan de Dios (1837-1937). Real Academia de Medicina y Cirugía de Murcia. Murcia: 1999.

10. Nelsone T, Durrheim DN, Speare R, Kiedrynski T, Melrose WD. Short communication: Strengthening sub-national communicable disease surveillance in a remote Pacific Island country by adapting a successful African outbreak surveillance model. Trop Med Int Health. 2006 Jan;11(1):17-21

11. Rankin J. Godzilla in the corridor: The Ontario SARS crisis in historical perspective. Intensive Crit Care Nurs. 2006 Jun;22(3):130-7. Epub 2005 Dec 1.

12. Durrheim DN, Harris BN, Speare R, Billighurst K. The use of hospital-based nurses for the surveillance of potential disease outbreaks. Bull World Health Organ. 2001;79(1):22-7. Epub 2003 Nov 5

13. Paugam H. Cholera++ epidemic in Kenya. Can Nurse. 1999 Mar;95(3):42-6

14. Pérez Picazo, M.T., Oligarquía urbana y campesinado en Murcia: 1875-1902. Biblioteca Murciana de bolsillo-Comunidad Autónoma de la Región de Murcia. Murcia: 1986.

15. Martínez Carrión, J. M. Historia Económica de la Región de Murcia. Siglos XIX y XX. Editora Regional.Murcia: 2002.

16. Valdespino-Gómez JL, Isibasi-Araujo A, Hinojosa-Ahumada MA, Giono-Cerezo S. Perspectiva de las vacunas contra el cólera. Salud Pública Mex. 1993;35:3-19. Disponible en: $\quad$ http://www.insp.mx/rsp/ files/File/1993/ene feb/ene fen 93 02perspectivas.pdf Consultado el: 21/5/10.

17. Germán Bes, Concha. Historia de la Enfermería Comunitaria I. De enfermeras visitadoras a instructoras sanitarias. Temperamentvm 2008, 8. Disponible en: http://www.index-f.com/temperamentum/tn8/t0608.php Consultado el: 23/06/2010

18. García Hourcade, J.J., Irigoyen López, A., García Olmo, M.A. Los Hospitales de la Diócesis de Cartagena en la documentación vaticana (visitas ad limina S.XVII - S. XIX). Disponible en: http://www.regmurcia.com/docs/murgetana/N104/N104 004.pdf Consultado el: 21/06/2010.

19. Velandia AL. Investigación histórica en enfermería. Conferencia central del taller "Investigación sobre historia de Enfermería". Coloquio Nacional de investigación en Enfermería. Medellín: 1997.

20. Velandia AL. Análisis de la priorización utilizada en la historia de la enfermería. Invest Educ Enferm. 2008; 26(1):36-47.

21. Siles J. Historia de la Enfermería. Editorial aguaclara. Segunda Edición. Alicante: 2008.

22. Ventosa F. Historia de la Enfermería Española. Editorial Ciencia. Madrid: 1984.

23. Herrera F, Lasante JE, Siles J. La edad contemporánea. El proceso de tecnificación e institucionalización profesional. En: Hernández F (Coord). Historia de la Enfermería en España (desde la antigüedad hasta nuestros días). Editorial Síntesis. Madrid: 1996.

24. Vázquez JM. La prensa escrita y la construcción de la realidad social. Disponible en: http://huespedes.cica.es/aliens/gittcus/licsr.htm Consultado el: 21/06/2010.

25. Rodríguez M. Historia General de Murcia. Almazura y Tres Fronteras Ediciones. Murcia: 2008. 
26. García B. Mariano Ruiz Funes: Lo cultural abre los poros de lo político. Comunicación del I Encuentro de jóvenes investigadores en historia contemporánea de la AHC. Disponible en: http://ifc.dpz.es/recursos/publicaciones/27/15/36.beatrizgarciaarce.pdf. Consultado el: 02/06/10.

27. Arroyo MS. La prensa murciana en el sexenio revolucionario (1868-1874). Anales de historia contemporánea. 3. 1993.

28. Jover MA. Archivos y documentación local en la región de Murcia. Documentación en Ciencias de la Información. 1990 (13). 113-131

29. Gabaldón Bravo EM, Siles González J, Cibanal Juan L, Domínguez Santamaría M, Vizcaya Moreno F. La situación social de la juventud en el área del cuidado a través de la prensa escrita. La década de 1868-1878 en Alicante (España). Index de Enfermería [Index Enferm] (edición digital) 2002; 36-37. Disponible en http://www.index-f.com/indexenfermeria/36-37revista/36-37 articulo 12-14.php

30. Siles González J. Praxis de la historia. La utilidad de la Enfermería en el desarrollo social, científico y profesional. Híades, 2000; 7: 55-89

31. Martín A. El PAPPS y el grupo español sobre promoción de la salud y prevención de la enfermedad. Aten Primaria. 2007 nov. 39(3):1

32. Leno D, Leno JL, Lozano MJ. Control de síntomas y tratamiento del cólera durante las primeras epidemias del siglo XIX. Metas Enferm. 2008 oct.11(8):22-26.

33. Oter C. La muerte y la institucionalización de los profesionales sanitarios. Educare21 2004; 13. Disponible en:

http://enfermeria21.com/educare/educare13/ensenando/ensenando3.htm

34. M. Elena Barros, Alicia Fernández, Mariola Fueyo y Elena Menéndez Atención domiciliaria en Gijón desde 1882 hasta nuestros días. Revista Hiades 8 (p. 431)

\section{BIBLIOGRAFÍA CONSULTADA}

Abad JM, Amorós F, Martínez MR. La Epidemia de cólera de 1834 en Archena y el Valle de Ricote. En Harrison: Principios de medicina interna. Madrid: 2005.

Estrella Sevilla, E. El sistema de saneamiento de la ciudad. Las Epidemias. En: Estrella Sevilla, E. Murcia: ciudad, territorio, cultura y agua. Ed. CPD Contraste S.L. Murcia: 2005.

García MJ. Manual para el uso de practicantes. Hiades. 7. Sept 2000.

Hernández J. Historia de la Enfermería. Un análisis histórico de los cuidados de enfermería. McGraw-Hill-Interamericana. Madrid: 1995.

OMS. Cólera. Disponible en http://www.who.int/topics/cholera/es/ Consultado el: 01/06/10.

Vera Nicolás, P. (Coord.) El Abastecimiento de agua potable en la Región. En: Murcia y el agua: Historia de una pasión. La Verdad Digital S.L. Disponible en: http://servicios.laverdad.es/murcia agua/cap14.htm Consultado el: 02/05/2010.

\section{REFERENCIAS “DIARIO DE MURCIA”}

(i) Días: 17/06/1885, 21/06/1885, 27/06/1885, 30/06/1885, 30/06/1885, 11/07/1885, 16/07/1885, 22/07/1885, 02/08/1885, 06/08/1885,

(ii) Día: $17 / 06 / 1885$

(iii) Día: 16/07/1885

(iv) Día: 27/06/1885, 11/07/1885

(v) Día: 21/06/1885. 30/06/1885

(vi) Día: 22/07/1885

(vii) Día: 30/06/1885

(viii) Día: 02/08/1885

(ix) Día: 06/08/1885 
(x) Día: $16 / 06 / 1885$

(xi) Día: $17 / 06 / 1885$

(xii) Días: $21 / 06 / 1885,09 / 07 / 1885$

(xiii) Día: $18 / 07 / 1885$

(xiv) Día: 03/07/1885

(xv) Día: $14 / 07 / 1885$

(xvi) Día: $10 / 07 / 1885$

(xvii) Día: $18 / 07 / 1885$

(xviii) Día: 01/08/1885

(xix) Días: 19/06/1885, 21/06/1885, 23/06/1885, 24/06/1885, 25/06/1885, 27/06/1885, 28/06/1885, 30/06/1885, 03/07/1885, 5/07/1885, 7/07/1885, 7/07/1885, 9/07/1885, 14/07/1885, 19/07/1885, 28/07/1885.

(xx) Días: 21/06/1885, 23/06/1885, 24/06/1885, 3/07/1885, 7/07/1885, 28/07/1885

(xxi) Día: $2 / 08 / 1885$

(xxii) Día: $7 / 08 / 1885$

(xxiii) Día: 30/07/1885

(xxiv) Día: $1 / 07 / 1885$

(xxv) Día: 8/07/1885

(xvi) Día: 10/07/1885

(xvii) Día: $18 / 07 / 1885$

(xviii) Día: 01/08/1885

(xxvi) Día: 20/06/1885

(xxvii) Día: 03/07/1885

(xxviii) Día: 26/06/1885

(xxix) Día: $16 / 06 / 1885$

(xxx) Día: 21/06/1885

(xxxi) Día: 27/06/1885

(xxxii) Dias: 18/06/1885, 05/07/1885, 09/07/188 